\title{
Comunicación
}

\section{Determinación de la Seroprevalencia del Virus de la Leucemia Bovina en un Establo Lechero de Lima, Perú}

\author{
Determination of the Seroprevalence of Bovine Leukemia Virus in a \\ Dairy Farm of Lima, Peru
}

Rocío Sandoval M. ${ }^{1.3}$, Alfredo Delgado C. ${ }^{1}$, Luis Ruiz G. ${ }^{1}$, Olger Ramos C. ${ }^{2}$

\section{Resumen}

\begin{abstract}
El objetivo del presente estudio fue determinar la seroprevalencia del virus de la leucemia bovina en un establo lechero de una universidad pública de Lima, Perú, a través de la determinación de anticuerpos circulantes contra el virus. El establo se encontraba infectado, tal y como muchos otros establos lecheros de la zona. Se encontró una prevalencia de $92.7 \%$ (51/55), donde el 100, 97 y $60 \%$ de los animales mayores de 5 años, de 2 a 5 años y menores de 2 años fueron seropositivos, respectivamente. Se reconoce la importancia de iniciar un plan de control y erradicación que sirva como modelo para los establos comerciales del departamento de Lima.
\end{abstract}

Palabras clave: enzoótica; ganadería; infección; leucosis; virus

\section{Abstract}

The aim of this study was to determine the seroprevalence of the bovine leukemia virus in a dairy farm of a public university of Lima, Peru through the circulating antibodies against the virus. The disease is prevalent in the farm as in many other dairy farms in the area. The results showed a prevalence of $92.7 \%$ (51/55), where 100, 97, and $60 \%$ of animals older than 5 years old, from 2 to 5 years old, and less than 2 years old were

\footnotetext{
${ }^{1}$ Clínica de Animales Mayores, ${ }^{2}$ Fundo «El Taro», Facultad de Medicina Veterinaria, Universidad Nacional Mayor de San Marcos, Lima

${ }^{3}$ E-mail: rociossandovalm@gmail.com
} 
seropositive respectively. It is recognized the importance of initiating a control and eradication programme that can be used as a model for other commercial dairy farms in the Lima region.

Key words: enzootic; livestock; infection; leucosis; virus

\section{INTRODUCCIÓN}

La leucosis enzoótica bovina (LEB) es una enfermedad viral, crónica y contagiosa que predomina principalmente en la ganadería lechera. Es causada por el virus de la leucemia bovina (VLEB), que es un deltaretrovirus de la familia Retroviridae (Baruta et al., 2012; Choudhury et al., 2013), que afecta las células de la línea linfoide, principalmente linfocitos B (Tomita et al., 2013; Aida et al., 2014), aunque también posee capacidad de infectar otras células, como los linfocitos $\mathrm{T}$ y monocitos (Chamizo, 2005; Hagiwara et al., 2014).

El contagio puede ser horizontal, de animal a animal (Gutiérrez, 2010), o vertical, de madre a hijo (Hagiwara et al., 2014). Los animales portadores sin manifestación clínica son la principal fuente de contagio en los establos lecheros (Gutiérrez, 2010). El contagio ocurre a través de secreciones y fluidos biológicos como leche, sangre, calostro, secreción nasal, saliva, semen y orina, donde se pueden llegar a encontrar linfocitos infectados (Gutiérrez, 2010; Choudhury et al., 2013; Khamesipour et al., 2013). No obstante, la mayor proporción de linfocitos infectados se encuentran en la sangre, por lo que se deben tomar medidas de precaución durante las vacunaciones, cirugías, aplicación de inyectables, etc. (Gutiérrez, 2010); aunque en un estudio en Brasil solo se encontró relación entre la presencia de la enfermedad y la inseminación artificial (Costa et al., 2013). Otro de los factores de riesgo que han sido relacionados con la propagación de la enfermedad es la adquisición de animales de reemplazo (Benavides et al., 2013).
Mientras más elevada sea la prevalencia de la enfermedad en un establo lechero, más favorecida se ve la transmisión horizontal. Por otro lado, la transmisión vertical es de menor importancia, considerando que menos del $10 \%$ de los animales nacidos de madres portadoras se infectan por el virus (Gutiérrez, 2010).

Aunque los animales se pueden infectar con el VLB a cualquier edad, los linfosarcomas y la linfocitosis persistente se presentan después de tres años del contagio (Baruta et al., 2012). Normalmente las infecciones son subclínicas. Solo el $30-70 \%$ del ganado infectado desarrolla una linfocitosis persistente y el menos del $10 \%$ desarrolla tumores (Benavides et al., 2013; Cenuse et al., 2013; Sandev et al., 2013). Los signos clínicos dependen del lugar en que aparecen los tumores y pueden incluir desarreglos digestivos, inapetencia, pérdida de peso, debilidad general y, a veces, manifestaciones neurológicas. Los ganglios linfáticos superficiales pueden verse incrementados en tamaño, pudiéndose palpar bajo la piel o por examen rectal (Gutiérrez, 2010).

La LEB es una enfermedad altamente transmisible, que genera un importante impacto económico en la ganadería lechera. Las pérdidas económicas son debidas a muertes prematuras, remplazo deanimales enfermos y disminución de la producción láctea, así como por las restricciones de importación y exportación impuestas por algunos países (Benavides et al., 2013). No obstante, en un hato lechero en Chile con $30 \%$ de seroprevalencia no se encontraron efectos negativos sobre fertilidad, aborto o mastitis, 


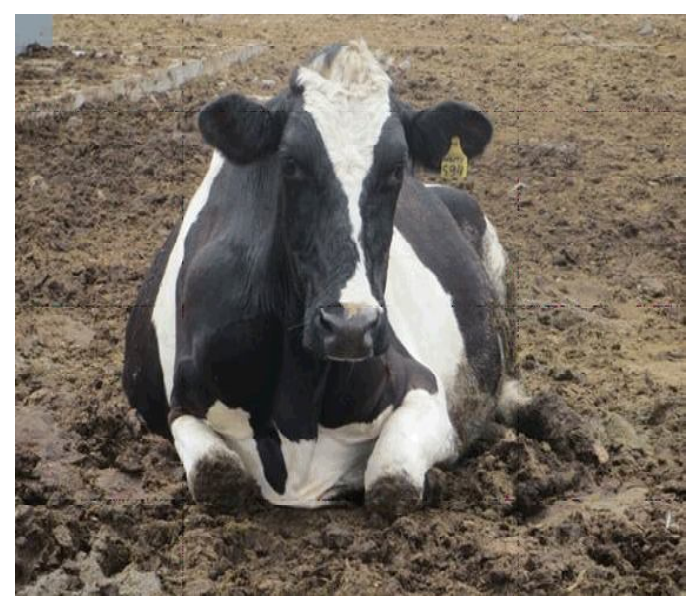

Figura 1. Vaca Holstein de 11 años con ganglios preescapulares incrementados de tamaño y fijos al tejido adyacente

pero se observó una pérdida promedio de 156 $\mathrm{kg}$ de leche por campaña por animal (Reinhardt et al., 1988). Por otro lado, en fincas lecheras de Canadá con seroprevalencias de $29 \%$ no se encontraron efectos adversos sobre la producción de leche, grasa o proteína (Tiwari et al., 2007); sin embargo, se estima que los costos por tratamientos y las pérdidas económicas equivalen a US\$ 800 al año para un hato de 50 vacas y con una prevalencia del 19\% (Chi et al., 2002).

En el Perú se dispone de escasa información sobre el estado actual de esta enfermedad, pero se sabe que es uno de los motivos de descarte en los establos lecheros de Lima (Orrego et al., 2003). Asimismo, en un estudio realizado en Arequipa se encontró una prevalencia de esta enfermedad de $12.8 \%$ (Flores y Rivera, 2000), siendo factible su erradicación. El objetivo del presente estudio fue estimar y establecer el nivel de infección a través de la determinación de anticuerpos circulantes contra el virus de la leucemia bovina en un establo lechero de una universidad pública, que, como la mayoría de los establos lecheros del departamento de Lima, presentan la enfermedad.

\section{Materiales y Métodos}

El estudio se realizó en octubre de 2013 en el establo docente de una universidad pública de Lima, Perú. Se hizo una revisión clínica a todos los animales en busca de lesiones compatibles con LEB. Asimismo, se hizo una revisión de los ganglios linfáticos prefemorales, preescapulares, submandibulares, supramamarios; además de una palpación rectal para evaluar los ganglios iliaco medio, iliofemorales y el útero.

Se seleccionaron animales con más de 6 meses de edad $(n=55)$ para evitar los anticuerpos calostrales. Se procedió a recolectar $3 \mathrm{ml}$ de sangre de la vena coccígea media o de la yugular empleando tubos al vacío sin anticoagulante. Se centrifugaron las muestras, se separó el suero resultante, se conservó a $4{ }^{\circ} \mathrm{C}$ y se procesó en el Laboratorio de Virología de la Facultad de Medicina Veterinaria de la Universidad Nacional Mayor de San Marcos, Lima.

Se realizó la prueba de ELISA para la detección de anticuerpos contra el VLEB, empleando un kit comercial (IDEXX Leukosis Serum X2 Ab Test), el cual detecta anticuerpos contra el antígeno de superficie gp51. Se siguieron los procedimientos sugeridos por el laboratorio fabricante del kit. Las lecturas espectrofotométricas fueron realizadas en un lector de ELISA (Bio-Tek Instruments, EEUU) a $450 \mathrm{~nm}$.

La prevalencia aparente fue estimada como la proporción de los animales infectados sobre el total de animales analizados. La prevalencia real fue estimada a partir de la prevalencia aparente, ajustada por la sensibilidad (95\%) y especificidad (97\%) diagnóstica de la prueba. Además, se determinó la prevalencia aparente en tres grupos etarios: menores de 2 años, de 2 a 5 años y mayores de 5 años. 


\section{Resultados}

En el examen clínico solo se encontró una vaca con los ganglios linfáticos incrementados de tamaño (Fig. 1); sin embargo, el $91 \%$ de los animales presentaron anticuerpos contra el VLEB (Cuadro 1). Asimismo, todos los animales mayores de 5 años y el $97 \%$ de los animales entre 2 y 5 fueron positivos al VLEB (Cuadro 2).

La prevalencia real, luego de corregir la prevalencia aparente por la sensibilidad y especificada de la prueba, resultó de $95.7 \%$.

\section{Discusión}

La alta prevalencia de VLB del establo se puede deber al alto grado de manipulación que reciben los animales por parte de los estudiantes y a la omisión de algunas prácticas de bioseguridad, como el uso de las mismas agujas en múltiples animales. Estudios de prevalencia en fincas lecheras de Brasil y Uruguay señalan que el 28 y $50 \%$ de los animales tuvieron contacto con el virus (Tomé et al., 2012; Costa et al., 2013), afectando al $87 \%$ de los hatos (Costa et al., 2013).

Prevalencias menores se encontraron en Nariño, Colombia (19.8\%), y Babol, Irán (8.5\%), según Benavides et al. (2013) y Hassanpour et al. (2014), respectivamente. No obstante, en estos y otros estudios, los casos clínicos con presencia de linfosarcomas o linfoma malignos fueron muy pocos o inexistentes (Benavides et al., 2013: Cenuse et al., 2013; Sandev et al., 2013).

Se han realizado una serie de intentos a nivel mundial para reducir la prevalencia del VLB en el ganado lechero. Los programas de control y erradicación de la LEB se basan en tres componentes: el primero consiste en identificar y eliminar animales positivos (Carignano et al., 2012; Lojkic et al., 2013), el segundo consiste en separar a los anima-
Cuadro 1. Detección de anticuerpos contra el virus de la leucemia en un establo lechero de Lima

\begin{tabular}{lcc}
\hline \multirow{2}{*}{ Diagnóstico } & \multicolumn{2}{c}{ Animales } \\
\cline { 2 - 3 } & $\mathrm{n}$ & $\%$ \\
\hline Positivo & 50 & 91 \\
Sospechoso & 1 & 2 \\
Negativo & 4 & 7 \\
\hline Total & 55 & 100 \\
\hline
\end{tabular}

Cuadro 2. Seroprevalencia de leucosis enzoótica bovina (LEB), según estrato etario, en un establo lechero de Lima

\begin{tabular}{ccc}
\hline $\begin{array}{c}\text { Grupo } \\
\text { etario } \\
\text { (años) }\end{array}$ & $\begin{array}{c}\text { Animales } \\
(\mathrm{n})\end{array}$ & $\begin{array}{c}\text { Positivos } \\
(\%)\end{array}$ \\
\hline$<2$ & 10 & 60 \\
2 a 5 & 26 & 97 \\
$>5$ & 19 & 100 \\
\hline
\end{tabular}

les positivos de los negativos (Rodríguez et al., 2011; Lojkic et al., 2013) y el tercero consiste en la aplicación de buenas prácticas sanitarias y bioseguridad con la finalidad de interrumpir el ciclo de transmisión, debido a que el virus provoca una infección persistente (Álvarez et al., 2012).

La eliminación del 95\% de los animales positivos en el establo del presente estudio sería inviable. La aplicación de esta estrategia ha sido muy exitosa en la erradicación de la LEB en varios países de Europa Occidental; sin embargo, los costos de la eliminación del ganado infectado superan las pérdidas económicas en establos con prevalencia superior al $12.5 \%$ (Chamizo, 2005) y, por lo tanto, son imprácticas, aunque podría justificar- 
se en establos con animales de pedigrí de alto valor genético, así como en ganaderías lecheras con fines de exportación de ganado a países «libres a BLV» (Rodríguez et al., 2011).

En lo que respecta a la separación de animales positivos de negativos, se recomienda una distancia mínima de 200 metros entre estos grupos, lo cual podría realizarse en el establo en estudio. Aunque este tipo de programa es bastante complicado y exigente, ha sido de gran utilidad para disminuir la prevalencia de la enfermedad en algunas ganaderías (Rodríguez et al., 2011; Âlvarez, et al., 2012), pero también ha fracasado en otras debido a los costos económicos, restricciones de gestión y carencia de una vacuna eficaz (Rodríguez et al., 2011). En un estudio realizado por Gutiérrez (2010), se halló que con la aplicación de estas medidas en un hato con $90 \%$ de seroprevalencia inicial, se pudo observar cambios significativos en la prevalencia luego de tres años; no obstante, no deja de ser una alternativa viable para limitar la propagación de la enfermedad.

Cualquiera que fuese el programa de control y erradicación a emplearse, se debe limitar la transferencia de las células infectadas con BLV a los animales libres de infección. Entre las principales prácticas se tiene: a) uso de agujas y jeringas descartables individuales, b) uso de guantes obstétricos descartables individuales, c) uso de material estéril en intervenciones quirúrgicas, tatuaje o aretado, d) administración de calostro de madres no infectadas, e) alimentación de los terneros con leche pasteurizada o con sustitutos, f) eliminación de vectores artrópodos, g) empleo de semen libre a VLB, h) prevenir la introducción de animales infectados, i) separación de animales por edad, j) minimizar el movimiento de animales, y k) limitar el acceso a los visitantes externos (Rodríguez et al., 2011).

Por otro lado, la segregación selectiva de los animales con un persistente elevado número de linfocitos en la sangre y serológicamente positivos al VLB sería una alternativa para el descarte de los principales diseminadores de la enfermedad (Baruta $e t$ al., 2012). Adicionalmente, Gutiérrez (2010) propone una segregación selectiva de los animales de acuerdo a su carga proviral en sangre periférica, interpretándola como un marcador del riesgo de transmisión de la infección. En una primera fase se pueden identificar a los bovinos con linfocitosis persistente y eliminarlos; asimismo, se debe realizar un control serológico periódico de todos los animales seronegativos mayores de seis meses, a fin de ir eliminando los positivos en la medida de las posibilidades del establecimiento.

En el establo en estudio se debe iniciar un proceso gradual de control y erradicación. Para esto, se debe desarrollar un programa de buenas prácticas sanitarias y bioseguridad, continuar con la identificación de los animales positivos antes de la toma de calostro, e implementar un banco de calostro proveniente de vacas negativas a leucosis. La prevalencia de este establo debe servir como una llamada de atención y alarma para la ganadería lechera en el Perú.

\section{Agradecimientos}

Los autores agradecen a los estudiantes del Taller de la Clínica de Animales Mayores de la UNMSM que participaron en el muestreo de los animales; en especial al Sr. Bernardo López, por su apoyo en la realización del proyecto. Asimismo, al Vicerrectorado de Investigación de la UNMSM por el financiamiento del estudio.

\section{Literatura Citada}

1. Aida Y, Takeshima S, Panei C, Omori T, Nunoya T, Davis W, et al. 2014. BLV-CoCoMo-qPCR: estimation of bovine leukemia virus (BLV) proviral load harbored by lymphocyte subpopulations in BLV-infected cattle at the subclinical stage of enzootic bovine 
leucosis. Retrovirology 11(Suppl 1): 143 (Abstr).

2. Álvarez I, Gutiérrez G, Martínez C, Politzki R, González C, Caviglia L, Trono K. 2012. Dinámica del virus de la leucosis bovina en un ciclo productivo de bovinos lecheros. En: Jornadas Latinoamericanas sobre Leucosis Bovina. Buenos Aires: Sociedad Argentina de Virología.

3. Baruta DA, Ardoino SM, Brandan JL, Sosa RE, Mariani EL, Riesco SR, Albrecht E. 2012. Relevamiento serológico de leucosis bovina enzoótica en tambos de tres departamentos de la zona norte de La Pampa. En: Jornadas Latinoamericanas sobre Leucosis Bovina. Buenos Aires: Sociedad Argentina de Virología.

4. Benavides B, Cedeño D, Serrano M. 2013. Epidemiological study of bovine leukemia virus in dairy cows in six herds in the municipality of Pasto, Nariño. Rev Lasallista Investig 10: 18-26.

5. Carignano H, Roldán D, Trono K, Miretti M, Poli M. 2012. Distribución de frecuencias alélicas del gen boladrb3 en animales blv(-) y blv(+) de baja carga proviral, pertenecientes a un rodeo comercial de raza Holstein y Holstein $x$ Jersey. En: Jornadas Latinoamericanas sobre Leucosis Bovina. Buenos Aires: Sociedad Argentina de Virología.

6. Cenuse C, Tirziu E, Nichita I, Cumpanasoiu C, Seres M. 2013. Hematological changes associated with enzootic bovine leukosis in cattle from Timis County. Lucrãri Ştiintifice 46: 26-30.

7. Chamizo E. 2005. Leucosis bovina enzootica. EDVET 6(7) [Internet], [06 mayo 2014]. Disponible en: http:// ww w. redal y c . or g/a r t i culo. oa? $\mathrm{id}=63612652016$

8. Chi J, VanLeeuwen J, Weersink A, Keefe G. 2002. Direct production losses and treatment costs from bovine viral diarrhoea virus, bovine leukosis virus, Mycobacterium avium subspecies paratuberculosis, and Neospora caninum. Prev Vet Med 55: 137-153.
9. Choudhury B, Finnegan C, Frossard J-P, Venables C, Steinbach F. 2013. Colostrum replacer and bovine leukemia virus seropositivity in calves. Emerg Infect Dis 19: 1027-1028.

10. Costa R, de Oliveira A, Salardane I, Ferreira P, Côgo R, Molinari D. 2013. Soroepidemiologia da leucose enzoótica bovina em propriedades leiteiras do município de Alegre, estado do Espírito Santo, Brasil. J Bras Ciên Anim 6: 427441.

11. Flores A, Rivera A. 2000. Seroprevalencia del virus de leucosis bovina en la cuenca lechera de Arequipa. Rev Inv Vet Perú 14: 144-148.

12. Gutiérrez G 2010. Estudio de la dinámica de infección perinatal con BLV en un rodeo de tambo de alta prevalencia. Tesis de Doctor. Argentina: Universidad de Buenos Aires. 165 p.

13. Hagiwara A, Saito M, Ishikawa Y, Kadota K. 2014. A histological study of lymphoid neoplasms in cattle infected with bovine leukosis virus. J Jpn Vet Med Assoc 67: 199-203.

14. Hassanpour M, Nadalian M, Safi S, Madani R, Bokaie S. 2014. Epidemiological survey of bovine leukemia virus (BLV) infection and its effective factors emphasis ELISA and nested PCR in dairy herds around Babol city (North of Iran) as a Caspian climate. Eur J Zool Res 3: 166-171.

15. Khamesipour F, Doosti A, Khodadoustan A, Goodarzi M. 2013. Molecular detection of bovine leukemia virus (BLV) in the frozen semen samples of bulls used for artificial insemination in Iran. ROAVS 3: 412-416.

16. Lojkic I, Balic D, Rudan N, Kovacic M, Cac Z, Periskic M, Bedekovic T, Roic B, Ciglar I. 2013. Eradication of bovine leukosis virus on a dairy farm through improved virus detection. Vet Arhiv 83: 581-591.

17. Orrego J, Delgado A, Echevarría L. 2003. Vida productiva y principales causas de descarte de vacas Holstein en la cuenca de Lima. Rev Inv Vet Perú 14: 68-73. 
18. Reinhardt G, Hochstein-Mintzel V, Riedemann S, Lealy H, Niedda M. 1988. Estudio serológico de leucosis enzoótica bovina en un predio de la provincia de Valdivia y su relación a parámetros productivos y reproductivos. J Vet Med B 35: 178-185.

19. Rodríguez S, Florins A, Gillet N, de Brogniez A, Sánchez-Alcaraz M, Boxus M, Boulanger F, et al. 2011. Preventive and therapeutic strategies for bovine leukemia virus: lessons for HTLV. Viruses 3: 1210-1248.

20. Sandev N, Zapryanova D, Stoycheva I, Susenova N, Mircheva T. 2013. Investigation of some haematological and blood biochemical parameters in cattle spontaneously infected with bovine leukosis virus. Mac Vet Rev 36: 107-110.
21. Tiwari A, VanLeeuwen J, Dohoo I, Keefe G, Haddad J, Tremblay R, Scott $H$, Whiting T. 2007. Production effects of pathogens causing bovine leukosis, bovine viral diarrhea, paratuberculosis, and neosporosis. J Dairy Sci 90: 659-669.

22. Tomé L, Obal G, Moratorio G, Bianchi $S$, Carrión F, Rama R, Lima A, et al. 2012. Análisis proteómico de partículas retrovirales purificadas del virus de laleucosis bovina. En: Jornadas Latinoamericanas sobre Leucosis Bovina. Buenos Aires: Sociedad Argentina de Virología.

23. Tomita K, Cyuzyo M, Kamomae Y, Yazima K, Uramoto K, Takeshima S, Aida Y. 2013. Investigation into the conditions and factors associated with the onset of bovine leucosis in Holstein cows in middle Hyogo prefecture. J Jpn Vet Med Assoc 